\title{
Duloxetine in the treatment of generalized anxiety disorder
}

\author{
Trevor R Norman \\ James S Olver
}

Department of Psychiatry, University of Melbourne, Austin Hospital, Heidelberg, Victoria, Australia
Correspondence:Trevor Norman Department of Psychiatry, University of Melbourne, Austin Hospital, Heidelberg, 3084,Victoria, Australia

Email trevorrn@unimelb.edu.au

\begin{abstract}
Duloxetine, a medication with effects on both serotonin and noradrenaline transporter molecules, has recently been approved for the treatment of generalized anxiety disorder. The evidence for its efficacy lies in a limited number of double blind, placebo controlled comparisons. Statistically significant improvements in the Hamilton Anxiety Rating Scale from baseline were demonstrated in all studies at doses of 60 to $120 \mathrm{mg}$ per day. The significance of such changes in terms of clinical improvements compared to placebo is less certain, particularly when the effect size of the change is calculated. In comparative trials with venlafaxine, duloxetine was as effective in providing relief of anxiety symptoms. In addition to improvements in clinical symptoms duloxetine has also been associated with restitution of role function as measured by disability scales. Duloxetine use is associated with nausea, dizziness, dry mouth, constipation, insomnia, somnolence, hyperhidrosis, decreased libido and vomiting. These treatment emergent side effects were generally of mild to moderate severity and were tolerated over time. Using a tapered withdrawal schedule over two weeks in the clinical trials, duloxetine was associated with only a mild withdrawal syndrome in up to about $30 \%$ of patients compared to about $17 \%$ in placebo treated patients. Duloxetine in doses of up to $200 \mathrm{mg}$ twice daily did not prolong the QTc interval in healthy volunteers. Like other agents with dual neurotransmitter actions duloxetine reduces the symptoms of generalized anxiety disorder in short term treatments. Further evidence for its efficacy and safety in long term treatment is required.
\end{abstract}

Keywords: duloxetine, generalized anxiety disorder, Hamilton anxiety rating scale, withdrawal syndrome, psycho-social function

\section{Introduction}

Generalized anxiety disorder (GAD) is a diagnosis of relatively recent origin (Tyrer and Baldwin 2006). The term anxiety neurosis, as defined by Freud, was used prior to 1980 to describe a condition characterized predominantly as feelings of unattached fearfulness. With the publication of the Diagnostic and Statistical Manual of Mental Disorders, Third Edition (DSM-III) anxiety neurosis was abandoned and GAD was introduced and distinguished from panic disorder. Under this rubric the diagnosis required that symptoms from three of four categories (motor tension, autonomic hyperactivity, apprehensive expectation, hyper-vigilance and scanning) to be present for at least 1 month. Under DSM-III GAD was essentially a residual diagnostic category. Modifications to the diagnostic schemata as in the publication of the revised edition of DSM-III (DSM-III-R), defined GAD as being characterized by chronic (more than 6 months), persistent, generalized anxiety not meeting criteria for panic disorder, phobic disorder or obsessive-compulsive disorder. Following evidence that GAD can exist independently of anxiety related to anticipation of a panic attack, exposure to a phobic stimulus or obsessional concern, DSM-III-R defined GAD by positive criteria related to unrealistic/excessive anxiety or worry about 2 or more life concerns and 6 of 18 physical symptoms including 9 autonomic symptom clusters. If another Axis I anxiety disorder was present, GAD could still be diagnosed if the 
anxiety/worry present was unrelated to it. DSM-IV further refined the diagnostic criteria of GAD by exclusion of autonomic symptoms from the list of somatic concerns required for diagnosis because of the low endorsement rate of these symptoms. The core features of the current definition of GAD revolve around uncontrollable and excessive worry. The worries experienced by patients with GAD reliably cluster around themes of family, finances, work and personal illness (Sanderson and Barlow 1990).

The alternate ICD-10 classification also recognises GAD as a separate disorder of chronic duration (6 months) but differs from DSM-IV with respect to the pervasiveness of the symptomatology. Thus ICD-10 does not require worry to be excessive or uncontrollable while there is an emphasis on autonomic hyper-arousal. Further a minimum of four symptoms from a list of 22 somatic symptoms must be present to meet diagnostic criteria.

The differences in diagnostic schemes lead to rather different estimates of prevalence depending on which one is used for diagnosis. Nevertheless various epidemiological studies, using the different criteria, suggest that GAD is a common psychiatric presentation with estimates of lifetime prevalence of $2.8 \%$ to $6.6 \%$ in the general population (Carter et al 2001; Kessler et al 2005). The 12-month prevalence of any anxiety disorder in the National Co-morbidity study conducted in 9,282 adults in the United States was 18.1\% (Kessler et al 2005). In this cohort the 12-month prevalence for GAD was $5.7 \%$. This figure is in reasonably close agreement to a number of epidemiological studies conducted in the European community (Lieb et al 2005). The lifetime prevalence of GAD ranged from $0.1 \%$ to $18.7 \%$, while 12 -month prevalence was reported as $0.1 \%$ to $2.1 \%$. Discrepancies between surveys can be accounted for by the different diagnostic criteria employed (DSM-III, DSM-IV or ICD-10) and the different instruments used to asses anxiety (CIDI or DIS in most studies). Where DSM-III criteria were employed prevalence is usually higher due to the shorter duration requirement. The antecedents of GAD are in all likelihood present in childhood but some studies suggest that the age of onset of the disorder is rare before the age of 20 years (Wittchen et al 1998; Lieb et al 2000). The majority of cases present for treatment in adulthood between 35 and 45 years of age (Wittchen et al 2000; Yonkers et al 2000; Carter et al 2001). Furthermore GAD has been reported to be the most common anxiety disorder in adults aged more than 55 years (Carter et al 2001).

Guidelines for the treatment of GAD recommend psychotherapy, pharmacotherapy or the combination of both (Anderson 2006). The NICE (National Institute of Clinical
Excellence, UK) guidelines indicate that psychotherapy should be first line treatment (NICE 2004). It is generally regarded that medication and psychotherapy are equally effective for acute treatment (Anderson 2006; Tyrer and Baldwin 2006). Indeed a meta-analysis of 35 studies showed that the effect size for cognitive behavior therapy (0.7) was similar to that for medications (0.6) (Gould et al 1997). Among the psychotherapies there is some evidence that cognitive behavior therapy has a better outcome at 6-month follow-up than anxiety management or analytical psychotherapy (Durham et al 1994). In longer term treatment psychological treatments might be better than medications but there are few comparative data. Improvement with cognitive behavior therapy can be maintained for up to 2 years (Kingdon et al 1996). In longer follow-ups (8-14 years), only about a third of patients make a good recovery (Durham et al 2003).

Considerations of the characteristics of GAD and the preferences of individual patients need to be taken into account when deciding treatments (Lam 2006). Particular issues pertaining to the clinical characteristics of GAD are the waxing and waning course of the illness, its chronicity and co-morbidity with other psychiatric and non-psychiatric disorders. Additionally prospective data suggest that GAD has a relatively low rate of remission. Thus in the Harvard-Brown Anxiety Research program (HARP) the probability of remission was 0.38 at 5 years (Yonkers et al 2000). In the 5-year follow-up period $38 \%$ of patients achieved a full remission, ie, a consecutive 8-week period with no or only occasional symptoms. The prognosis must be regarded as unfavorable.

Patients with GAD commonly present with a co-existing depressive disorder (Baldwin and Polkinghorn 2005). Indeed based on the results of the National Co-morbidity Survey (Kessler et al 1994) it might be stated that co-morbidity is the rule rather than the exception. For GAD the total lifetime co-morbidity with another psychiatric diagnosis was $90.4 \%$ and the 30-day co-morbidity was $66.3 \%$ with the following disorders: mania, major depression, dysthymia, panic disorder and phobic disorders, alcohol or drug abuse (Wittchen et al 1994). This high co-morbidity has been used to suggest that GAD may be a residual or prodrome of another disorder (Wittchen et al 1994). On the other hand about a third of subjects with a current diagnosis of GAD did not have any other recent diagnosis in the National Co-morbidity study, which argued for GAD as an independent diagnostic category (Wittchen et al 1994).

For the short term treatment of GAD benzodiazepines have been shown to be more effective than placebo (Shader and Greenblatt 1993; Ballenger 2001; Davidson 2001; Baldwin 
and Polkhinghorn 2005). A statistically significant effect size (Cohen's $d=0.38 \pm 0.15 ; \mathrm{p}<0.0001$ ) was noted for the benzodiazepines compared to placebo in the treatment of GAD using meta-analysis (Hidalgo et al 2007). However, concerns about their long term use, particularly issues of tolerance and dependence, have seen a general reluctance to use benzodiazepines in clinical practice (Schweizer and Rickels 1998). Antidepressant medications have been extensively used in the treatment of various anxiety disorders and the evidence for their efficacy in panic disorder, obsessive compulsive disorder and to a lesser extent in social anxiety disorder is well documented (Gorman 2002). More recently the effectiveness of antidepressants in GAD has been evaluated in a number of double-blind, placebo controlled clinical trials. The efficacy of the selective serotonin reuptake inhibitors (SSRIs) in GAD has been frequently canvassed (Baldwin and Polkinghorn 2005). Beyond the SSRIs interest has been shown in so called "dual acting" antidepressants, most recently duloxetine.

\section{Pharmacolgy of duloxetine Mechanism of action}

Duloxetine $((+)-(\mathrm{S})-\mathrm{N}$-methyl- $\gamma-(1-$ naphthalenyloxy $)-$ 2-thiophene-propanamine) inhibits serotonin (5HT) and noradrenaline (NE) reuptake into nerve endings or slice preparations with the NE/5HT ratio between 2 and 9 both in vivo and in vitro (Kasamo et al 1996; Wong 1998). The drug has a high affinity for human cloned serotonin and noradrenaline transporters in vitro and is more potent than venlafaxine (Bymaster et al 2001). Duloxetine has low affinity for a range of other serotonin, muscarinic, adrenergic and histaminergic receptors and does not significantly inhibit monoamine oxidase A or B activity (Bymaster et al 2001). Microdialysis studies of acute doses showed that duloxetine significantly enhanced the release of extra-cellular levels of both serotonin and noradrenaline in the pre-frontal cortex and hypothalamus of drug naïve rats (Engelman et al 1995). On the other hand, an in vitro study in healthy volunteers are at odds with the animal data and suggest that at doses of 20 and $60 \mathrm{mg} /$ day duloxetine was selective for serotonin reuptake inhibition (Turcotte et al 2001). At higher doses (80 and $120 \mathrm{mg} /$ day) an effect on noradrenaline reuptake was evident (Vincent et al 2004). The pharmacology of duloxetine implies that it is the effect on both serotonin and noradrenaline reuptake which may account for the antidepressant activity of the drug.

\section{Pharmacokinetics}

Duloxetine pharmacokinetics was determined after single and multiple oral doses in healthy volunteers. Duloxetine is administered as an enteric coated pellet formulation due to its acid lability (Bymaster et al 2005). The drug exhibited linear pharmacokinetics in healthy male volunteers who received $20 \mathrm{mg}$ twice daily with a dose escalation at weekly intervals to 30 and $40 \mathrm{mg}$ twice daily (Sharma et al 2000). Steady state plasma concentrations were attained within 3 days for each dosing regimen in this study. The effect of food on the rate of absorption of duloxetine was investigated in 12 healthy female volunteers following a single oral dose of $40 \mathrm{mg}$ (Skinner et al 2000). The subjects received the drug on 4 separate occasions separated by one week: after an overnight fast; 15 minutes after a high fat meal; at bedtime and again after an overnight fast. Both food and night time administration delayed the time to maximum concentration (by a mean of 4 hours) but only bedtime administration reduced the extent of absorption (AUC decreased $\sim 18 \%$ ). The effects of food were considered to be relatively modest. A median 2-hour lag time was apparent until absorption began $\left(\mathrm{T}_{\text {lag }}\right)$, with maximal plasma concentrations ( $\mathrm{Cmax}$ ) of duloxetine occurring about 6 hours after the dose. The apparent volume of distribution of duloxetine is large. On repeated dosing the mean apparent volume of distribution for duloxetine was $1943 \mathrm{~L}$ with a range of 803 to $3531 \mathrm{~L}$ (Sharma et al 2000). In this study the mean apparent oral clearance was $114 \mathrm{~L} / \mathrm{h}$ with a range of 44 to $218 \mathrm{~L} / \mathrm{h}$ (Sharma et al 2000). The data in young and elderly females after a single oral dose of $40 \mathrm{mg}$ agree reasonably well with the data obtained for males (Skinner et al 2003). Thus the mean apparent clearance was $70.3 \mathrm{~L} / \mathrm{h}$ and the mean apparent volume of distribution was $962 \mathrm{~L}$ in younger women (32-50 years). Age had no statistically significant effect on these parameters in elderly women (65-77 years) with a mean clearance $52.9 \mathrm{~L} / \mathrm{h}$ and mean volume of distribution $1077.9 \mathrm{~L}$ (Skinner et al 2003). Duloxetine is highly bound (>90\%) to proteins in human plasma, binding primarily to albumin and $\alpha_{1}$-acid glycoprotein. The interaction between duloxetine and other highly protein bound drugs has not been fully evaluated. Plasma protein binding of duloxetine is not affected by renal or hepatic impairment. The mean elimination half-life of duloxetine is about 12 hours (range 7-27 hours) (Sharma et al 2000; Skinner et al 2003). Following single oral doses of 20,40 or $60 \mathrm{mg}$ and multiple oral doses of 20 or $40 \mathrm{mg}$ for 5 days there were no clinically significant differences in the pharmacokinetics of Japanese and Caucasian subjects (Chan et al 2007). Similarly a retrospective comparison of pharmacokinetic parameters obtained after single and multiple oral dosing with $60 \mathrm{mg}$ in healthy Chinese volunteers were consistent with the data obtained for Japanese and Caucasian subjects (Tianmei et al 2007). 
Elimination of duloxetine is mainly through hepatic metabolism involving two P450 isozymes, CYP2D6 and CYP1A2. Biotransformation and disposition of duloxetine has been determined following oral administration of ${ }^{14} \mathrm{C}$-labeled duloxetine (Lantz et al 2003). Unchanged duloxetine comprised about $3 \%$ of the total radio-labeled material in plasma, indicating that it underwent extensive metabolism to numerous metabolites. The major biotransformation pathways for duloxetine involve oxidation of the naphthyl ring followed by conjugation and further oxidation. Both CYP2D6 and CYP1A2 catalyze the oxidation of the naphthyl ring in vitro. Metabolites found in plasma include 4-hydroxy duloxetine glucuronide and 5-hydroxy, 6-methoxy duloxetine sulphate. Many additional metabolites have been identified in urine, some representing only minor pathways of elimination. Only trace $(<1 \%$ of the dose) amounts of unchanged duloxetine are present in the urine. Most (about $70 \%$ ) of the duloxetine dose appears in the urine as metabolites of duloxetine; about $20 \%$ is excreted in the feces (Lantz et al 2003). As would be expected for a drug extensively metabolized by the liver, patients with hepatic impairment arising in the context of cirrhosis show alterations in single dose pharmacokinetics (Suri et al 2005). Following a single oral dose of $20 \mathrm{mg}$ apparent plasma clearance was lower (24 versus $160 \mathrm{~L} / \mathrm{h}$ ) and systemic exposure (as measured by AUC values) was higher (775 versus $268 \mathrm{ng} / \mathrm{h} / \mathrm{mL}$ ) in the cirrhotic patients compared to healthy controls. For these same subjects mean elimination half life was 3 times longer (47.8 versus $13.5 \mathrm{~h}$ ) in cirrhotic patients compared to healthy volunteers.

\section{Use of duloxetine in generalized anxiety disorder \\ Pre-clinical data}

Evidence for the use of antidepressants in the treatment of anxiety disorders, at least based on the available animal models, is not well supported (Borsini et al 2002). Many of the models are established on their ability to detect the activity of the benzodiazepines and may not be representative for other classes of agents. In the case of antidepressants two factors may mitigate against the chances of positive results: clinical effects are often observed with chronic administration whereas the animal models for the most part rely on acute effects; many antidepressants, at least initially, are anxiogenic. Pre-clinical evidence for the anxiolytic effect of duloxetine has been observed after chronic administration in one animal model of anxiety (Troelsen et al 2005). The effects of 21 days treatment with $10 \mathrm{mg} / \mathrm{kg}$ twice daily of duloxetine, citalopram, reboxetine and amitriptyline in the zero maze were compared to their effects after acute administration. None of the agents was anxiolytic in this test after acute doses and indeed there was evidence for anxiogenic effects of reboxetine, duloxetine and amitriptyline. Only chronic duloxetine administration produced an anxiolytic response dissociable from non-specific motor effects in this task. The anxiolytic effect was attributed to the reduction in the serotonin transporter protein in the cortex of mice treated with duloxetine.

\section{Clinical data}

Duloxetine was approved for the treatment of GAD by both the US Federal Drug Administration and the British authorities early in 2007. Despite this approval there are relatively little published data to support its use in this condition, although a priori it would be expected to be effective given similar data for other "dual acting" compounds such as venlafaxine. It has been speculated that all SNRI antidepressants (venlafaxine, duloxetine, milnacipran) might have similar efficacy across a range of conditions including depression, chronic pain, PTSD, panic disorder and social anxiety disorder (Stahl et al 2005; Baldwin 2006).

Among the first indications that duloxetine might be suitable for the treatment of anxiety symptoms was an analysis of its effect in trials of major depressive disorder (Dunner et al 2003). Four placebo controlled trials were evaluated and its potential anxiolytic effect assessed from the changes on the anxiety/somatization factor and psychic anxiety (item 10) of the Hamilton Depression Rating Scale (HAM-D). In two of the studies (Goldstein et al 2002; Goldstein et al 2004), data from the Hamilton Anxiety Rating Scale (HAM-A) were also available. Within the limitations of the data set (exclusion of patients with primary anxiety disorder in past 12 months) duloxetine was more effective than placebo in alleviating anxiety symptoms in these depressed patients. The dose of duloxetine ranged from 40 to $120 \mathrm{mg}$ /day. The study could not assess whether the effect on anxiety symptoms occurred independently of the effect on the symptoms of depression. In this pooled comparison duloxetine was also noted to be more effective than either of the comparator agents, paroxetine and fluoxetine. For both comparator agents the dose was fixed and the number of patients was smaller than the total number of patients treated with either duloxetine or placebo. The possibility that the result arises because of a statistical artefact cannot be overlooked.

Recent clinical trials have investigated the efficacy of duloxetine for the treatment of patients with GAD. Each of the 
studies used similar diagnostic criteria (DSM-IV determined using the Mini International Neuropsychiatric Interview) to ensure that patients met criteria for GAD only. In particular patients were excluded if they met criteria for a recent (within the past 6 months) Major Depressive Disorder or substance abuse. A history within the past year of panic disorder, posttraumatic stress disorder or eating disorder was also an exclusion criterion. Thus patients who were included in the study may have had a lifetime co-morbidity of other mood disorders with GAD but not a current co-morbidity. A lifetime history of psychotic, bipolar or obsessive compulsive disorders was an additional diagnostic exclusion criterion. To ensure that anxiety symptoms predominated the clinical picture patients were required to have a Covi Anxiety score $(\mathrm{CAS})>9$ and no item in the Raskin Depression Scale $>3$ at baseline. Furthermore patients with an Axis II diagnosis were excluded from the studies. The studies and their main findings are summarized in Table 1.

Duloxetine was compared to placebo in a 9-week, doubleblind, flexible dose study in 513 patients meeting DSM-IV criteria for GAD (Koponen et al 2007). After a screening and washout phase of up to 30 days patients entered a single-blind, placebo phase of 1 week. An acute therapy phase of 9 weeks followed together with a 2 -week discontinuation phase. Patients were randomly assigned to treatment with $60 \mathrm{mg}$ once daily, $120 \mathrm{mg}$ once daily duloxetine or placebo. The total HAM-A score was used in the assessment of efficacy but did not form part of the entry criteria. However patients were stratified according to HAM-A total scores $<$ or $\geq 22$ and were recruited from 42 outpatient clinics in 7 countries. Study visits were conducted at weeks 1, 2, 4, 6 and 9 of the double-blind treatment phase. Assessment of the efficacy of treatments was based on the change in the total HAM-A score from baseline in addition to a number of secondary parameters: HAM-A psychic and somatic factors; Hospital Anxiety and Depression Scale (HADS); Patient and Clinician Global Impression-Improvements scales; Sheehan Disability Scale. For continuous efficacy variables an analysis of covariance was used to assess change from baseline. In addition a mixed effects repeated measures analysis was performed to assess change over time. Statistically significantly greater differences were observed for the reduction in anxiety symptoms in patients treated with either dose of duloxetine than for placebo ( $p<0.001$ ). Differences in mean HAM-A scores from baseline were $>4$ points larger in duloxetine than in placebo treated patients. In this study the effect size could not be calculated as the variance in the HAM-A change scores was not reported. Based on a 50\% reduction in HAM-A score from baseline, response rates were $58 \%$ for duloxetine $60 \mathrm{mg}$, $56 \%$ for duloxetine $120 \mathrm{mg}$ and $31 \%$ for placebo $(\mathrm{p}<0.001)$. Remission was defined as achieving a HAM-A score $\leq 7$ at endpoint. Based on this criterion $31 \%$ of duloxetine $60 \mathrm{mg}$, $38 \%$ duloxetine $120 \mathrm{mg}$ and $19 \%$ of placebo treated patients achieved remission. There did not appear to be any clinical

Table I Efficacy of duloxetine in the treatment of generalized anxiety disorder: double-blind, placebo-controlled studies

\begin{tabular}{|c|c|c|c|c|c|c|}
\hline \multirow[t]{2}{*}{ Study } & \multirow[t]{2}{*}{ Treatment } & \multirow{2}{*}{$\begin{array}{l}\text { Duration of } \\
\text { treatment }\end{array}$} & \multirow{2}{*}{$\begin{array}{l}\text { Primary* } \\
\text { outcome }\end{array}$} & \multicolumn{3}{|c|}{ Response rates** } \\
\hline & & & & DUL & VEN & PBO \\
\hline \multirow[t]{3}{*}{ Koponen et al 2007} & 60 mg/day DUL & 9 weeks & $-12.8^{\dagger}$ & $58 \%$ & & $31 \%$ \\
\hline & 120 mg/day DUL & & -12.5 & $56 \%$ & & \\
\hline & PBO & & -8.4 & & & \\
\hline \multirow[t]{3}{*}{ Hartford et al 2007} & $60-120 \mathrm{mg} /$ day DUL & 10 weeks & $-11.8(0.69)$ & $47 \%$ & $54 \%$ & $37 \%$ \\
\hline & 75-225 mg/day VEN & & $-12.4(0.67)$ & & & \\
\hline & PBO & & $-9.2(0.67)$ & & & \\
\hline \multirow[t]{2}{*}{ Rynn et al 2007} & 60-120 mg/day DUL & 10 weeks & $-8 . I^{\dagger}$ & $40 \%$ & & $32 \%$ \\
\hline & PBO & & -5.9 & & & \\
\hline \multirow[t]{4}{*}{ Nicolini et al 2008} & 20 mg/day DUL & 10 weeks & $-14.7(1.0)$ & $60 \%$ & $61 \%$ & $42 \%$ \\
\hline & $60-120 \mathrm{mg} /$ day DUL & & $-15.3(0.7)$ & $65 \%$ & & \\
\hline & 75-225 mg/day VEN & & $-15.5(0.7)$ & & & \\
\hline & PBO & & $-11.6(0.7)$ & & & \\
\hline
\end{tabular}

*Mean change from baseline for the HAM-A score (SEM).

$* *$ Reduction in the baseline HAM-A score $\geq 50 \%$.

†Variance measure not reported.

Abbreviations: DUL, duloxetine; PBO, placebo;VEN, venlafaxine. 
advantage for the higher dose of duloxetine, in terms of remission, over the lower dose. Statistically there were no differences between the two duloxetine doses for any of the efficacy measures used. In all secondary efficacy variables duloxetine 60 and $120 \mathrm{mg}$ were superior to placebo statistically. Greater improvements in functioning were observed in both duloxetine groups compared to placebo $(p<0.001)$ based on the change from baseline in the Sheehan Disability Scales. The data suggest that duloxetine attenuated the symptoms of GAD.

A similar double-blind, placebo-controlled trial of duloxetine was conducted in patients with a DSM-IV diagnosis of GAD over a 10-week period (Rynn et al 2007). Patients were recruited from 27 outpatient treatment centres in the US. To be eligible for the study, in addition to meeting DSM criteria, patients also had to meet certain severity criteria: Clinical Global Impression-Severity Scale (CGI-S) $\geq 4$; Hospital Anxiety and Depression Scale (HADS) anxiety subscale $\geq 10$; CAS $\geq 9$; and CAS total $>$ Raskin Depression Scale. After a screening and washout phase of up to 30 days patients entered a single-blind, placebo phase of 1 week. An acute therapy phase of 10 weeks followed together with a 2-week discontinuation phase. The primary efficacy measure was the HAM-A total score administered at each visit (weeks 1, 2, 4, 7 and 10). Secondary outcomes focused on improvements in overall symptom severity and global functioning. A flexible dosing schedule was adopted, such that a maximum of $120 \mathrm{mg}$ /day of duloxetine could be attained. The primary efficacy variable was the mean change from baseline to endpoint in the total HAMA-A score. Statistical differences were assessed using analysis of co-variance, while change over time was assessed with a mixed-effects repeated measures method. A total of 168 patients treated with duloxetine and 159 treated with placebo were included in the statistical analyses. A statistically significant difference between duloxetine and placebo was evident from week 2 of treatment ( $p<0.001)$. Statistically significant differences were evident at endpoint for the change in total HAM-A scores observed in duloxetine (8.12) and placebo (5.89) treated patients $(p<0.05)$. Similar or greater differences were observed for the HAM-A factors and items. It was not possible to calculate an effect size for this study as the variance in the change scores was not reported. Based on the mean changes in the HAM-A score compared to that of the previous study, the effect of duloxetine did not appear to be as robust. Response, defined as a $\geq 50 \%$ reduction in HAM-A score from baseline, was $40 \%$ for duloxetine and $32 \%$ for placebo $(p<0.05)$. However for remission, a HAM-A score at endpoint $\leq 7$, there was no statistically significant difference between duloxetine (28\%) and placebo (23\%). The mean final dose of duloxetine at endpoint was $101.9 \mathrm{mg}$ /day with the majority of patients (58\%) achieving $120 \mathrm{mg} /$ day. Despite the opportunity in this study to examine dose response effects for duloxetine such an analysis was not undertaken. The study suggests that duloxetine may possess a modest efficacy in the short term treatment of GAD.

Duloxetine 60 to $120 \mathrm{mg}$ /day was compared to venlafaxine XR (75-225 mg/day) and placebo over 10 weeks in adult patients meeting the DSM-IV criteria for the disorder (Hartford et al 2007). Almost 500 patients were randomly assigned to duloxetine $(n=160)$, venlafaxine XR $(n=164)$ or placebo $(n=161)$ and the efficacy was assessed by the change in the HAM-A Rating score from baseline to end point. For the evaluation of statistical significance of change an analysis of covariance was used. Greater improvement was observed in both the duloxetine $(\mathrm{p}<0.01)$ and venlafaxine $(\mathrm{p}<0.001)$ groups than for the placebo treated patients. There were no statistically significant differences between the duloxetine and venlafaxine groups. Cohen's d was used to compute an effect for this trial based on the reported mean (and standard deviation) change from baseline in the HAM-A scores for patients in the three treatment arms. For duloxetine 60 to $120 \mathrm{mg} /$ day compared to placebo the Cohen's d was 0.30 (95\% confidence interval [CI] 0.08 to 0.52 ) and for venlafaxine 72 to $225 \mathrm{mg}$ /day compared to placebo Cohen's d was calculated as 0.37 (95\% CI 0.15-0.59). These effect sizes are similar to those reported in a recent meta-analysis for antidepressant treatment of GAD (Hidalgo et al 2007). For the comparison of duloxetine and venlafaxine Cohen's $\mathrm{d}$ was 0.07 ( $95 \% \mathrm{CI}-0.15$ to 0.29 ), suggesting no difference in efficacy between the two drugs. Discontinuation of treatment for adverse events was greater in the duloxetine (14.2\%) and venlafaxine (11.0\%) groups than for placebo group (1.9\%). On the other hand the overall rate of discontinuation for any reason was not different for the three groups. Following tapering of the medication over a 2-week period at the end of the trial there were significantly more emergent adverse events for venlafaxine treated patients $(26.9 \%)$ than for either duloxetine (19.4\%) or for placebo (15.8\%). Duloxetine was equivalent to venlafaxine in the symptomatic relief of GAD and both provided greater relief than placebo.

A further comparative study of duloxetine and venlafaxine was performed in 33 non-US sites (Nicolini et al 2008). Diagnostic criteria were substantially the same as those used in the previous trials with the HAM-A scale as the primary efficacy measure. Secondary efficacy measures were assessed 
from the psychic and somatic anxiety factors scores of the HAM-A, Sheehan Disability Scale, HADS, CGI-I and the Patient Global Impression Improvement (PGI-I) scales. Patients completed a 3- to 30-day screening phase and then were randomly assigned to duloxetine $20 \mathrm{mg}$ once daily, duloxetine 60 to $120 \mathrm{mg}$ once daily, venlafaxine 75 to $225 \mathrm{mg}$ once daily or placebo. For the higher duloxetine dose group and for venlafaxine the dose was titrated according to response throughout the 10 -week treatment period. Statistical analyses were conducted on the intent to treat population. The primary efficacy analysis was the change from baseline in the HAM-A total score, which was compared between treatment groups using an analysis of covariance (ANCOVA) model with treatment and investigator as fixed effects and baseline score as a covariate. A mixed model repeated measures analysis was also performed. Based on the LOCF and mixed models analyses all three active treatment groups demonstrated a statistically significant difference from placebo in reducing the HAM-A total score (duloxetine $20 \mathrm{mg}$ versus placebo $\mathrm{p}<0.01$; duloxetine $60-120 \mathrm{mg}$ and venlafaxine versus placebo $\mathrm{p}<0.001)$. Similar results were also obtained for psychic and somatic anxiety factors. For effect size calculations Cohen's d was used. For the comparison of duloxetine $20 \mathrm{mg}$ and placebo d was 0.34 (95\% CI 0.08-0.61) and for duloxetine 60 to $120 \mathrm{mg}$ and placebo d was 0.42 (95\% CI $0.20-0.62$ ). Both effect sizes were similar to that which could be calculated for the comparison of venlafaxine and placebo $(\mathrm{d}=0.44 ; 95 \%$ CI $0.22-0.66)$. There were no differences between venlafaxine and duloxetine $20 \mathrm{mg}$ /day $(\mathrm{d}=0.09$; $95 \%$ CI -0.18 to 0.36 ) or duloxetine 60 to $120 \mathrm{mg} /$ day ( $d=0.02 ; 95 \% \mathrm{CI}-0.20$ to 0.25 ) based on the calculation of Cohen's d. Similarly duloxetine $20 \mathrm{mg}$ and duloxetine 60 to $120 \mathrm{mg}$ were not different $(\mathrm{d}=0.07 ; 95 \% \mathrm{CI}-0.20$ to 0.34). Again the study provides evidence for the efficacy of duloxetine in the treatment of GAD at least as effective as venlafaxine.
Duloxetine was compared to venlafaxine in adult patients with GAD using non-inferiority criteria (Allgulander et al 2008). In this report data from two previous trials (see Hartford et al 2007; Nicolini et al 2008) were combined and six noninferiority criteria were established by an independent (of the pharmaceutical manufacturer) panel of experts. The consensus panel's recommendations for non-inferiority are noted in Table 2. The first four criteria needed to be satisfied before the last two were considered. The statistical analysis used the HAM-A score as the primary outcome measure while the non-inferiority analyses used a lower bound of a one-sided $97.5 \%$ confidence interval for the difference between the test intervention and reference intervention as recommended by the International Committee on Harmonization (ICH 1998). The primary analysis was conducted on the per-protocol patients who were treated with duloxetine $(n=239)$ or venlafaxine XR $(n=262)$. The per-protocol sample was defined as patients who had completed at least 4 weeks of treatment, had baseline and post-baseline HAM-A ratings after at least 4 weeks of treatment, and did not have any protocol violations that were judged to potentially have an impact on the analysis or conclusions. Duloxetine 60 to $120 \mathrm{mg}$ /day met all statistical and clinical criteria for non-inferiority with a mean difference in HAM-A total score improvements between duloxetine and placebo of -3.8 , compared to the difference between venlafaxine XR and placebo of -3.6 points. Subtracting the two drug-placebo mean differences yielded a point estimate of 0.20 HAM-A total score points in favor of duloxetine. The lower bound of the confidence interval for this point estimate was -1.28 in the per-protocol sample which was within the pre-specified -1.5 margin. Thus the criterion for non-inferiority was met. Response rates for duloxetine, venlafaxine XR, and placebo were 56\%, 58\% and $40 \%$, respectively.

A further pooled analysis of data from the four clinical trials was performed to examine the efficacy and tolerability

Table 2 Non-inferiority criteria used for comparative analysis of duloxetine and venlafaxine in generalized anxiety disorder ${ }^{\mathrm{a}}$

I. At least one three-arm double-blind comparison trial for test intervention with an active comparator.

2. Both the test intervention and the active comparator should be superior to placebo by a clinically meaningful difference in the HAM-A total score (set at $\geq 2$ points by the panel).

3. Treatment response rates (defined as $\geq 50 \%$ reduction in HAM-A total score from baseline to study end point) for the test and active comparator groups should be at least 10 percentage points greater than the response for placebo.

4. Both the active and test intervention have to be statistically significantly better than placebo on the primary outcome measure.

5. The non-inferiority margin between the test intervention and the active comparator is $<50 \%$ of the difference between active comparator and placebo, and this difference is not clinically meaningful.

6. The response rate of the test intervention is not more than 5 percentage points lower than the response rate in the active comparator group.

aFrom the pooled non-inferiority comparison reported by Allgulander et al 2008. 
of duloxetine in elderly patients (Davidson et al 2008). From the database there were 73 patients ( 45 randomly assigned to duloxetine and 28 to placebo) older than 65 years. This represented a relatively small proportion (4.9\%) of the total population treated. Compared to placebo treated subjects, duloxetine treated patients had statistically significantly greater improvements for the HAM-A total score $(\mathrm{p}<0.05)$ and psychic $(\mathrm{p}<0.05)$ but not somatic anxiety factors. Based on the reported change scores from baseline to endpoint of the total HAM-A scores Cohen's d was calculated as 0.56 (95\% CI 0.04-1.02), which is within the range of that reported for other anxiolytic agents (Hidalgo et al 2007). In this patient population there were high discontinuation rates for adverse effects $(22.2 \%$ for duloxetine versus $0 \%$ for placebo). Evaluations in populations of elderly patients are of further interest, particularly given that GAD is reputedly the most common anxiety disorder in adults aged 55 years or more (Carter et al 2001).

Two pooled analyses of the placebo arms of the controlled trials were conducted to examine duloxetine efficacy and effect on functional outcomes. Patients assigned to the venlafaxine arm of the Hartford et al (2007) study were not included in the pooled analysis. The pooled analysis gave a database of 668 patients treated with duloxetine 60 to $120 \mathrm{mg} /$ day and 495 patients treated with placebo for up to 10 weeks. With respect to efficacy, duloxetine-treated patients improved statistically significantly more from baseline to endpoint than did placebo treated patients (11.1 points versus 8.0 points on the HAMA-A scale; $\mathrm{p}<0.001$; ANCOVA) (Allgulander et al 2007). The authors suggest that such improvements are clinically important representing a decline in baseline severity of anxiety by about a half. Remission of symptoms was attained by about a third of patients treated with duloxetine. Both psychic and somatic anxiety sub-scales of the HAM-A scale were improved. Using the data reported in this pooled analysis it was possible to calculate an effect size for baseline HAM-A scores as well as the treatment endpoint HAM-A scores. At baseline the effect size was 0.01 (Cohen's $d=0.02$ ). The groups were well matched and not significantly different. At end point the effect size was 0.18 (Cohen's $\mathrm{d}=0.37$ ). The effect of duloxetine in GAD can be regarded as relatively modest based on the pooled analysis particularly when compared to the effect sizes calculated in recent meta-analyses for antidepressants used in the treatment of GAD (Mitte et al 2005; Hidalgo et al 2007).

Along with the improvements in anxiety symptomatology self-reported assessments of psycho-social functioning were also significantly improved in a pooled analysis
(Endicott et al 2007). In each of the three studies the Sheehan Disability Scale (SDS), including the subscales global functioning, work/school life, social life and family home responsibility, were measured and showed a fall to mild severity in the duloxetine treated patients compared to moderate severity in the placebo treated patients. For duloxetine-treated patients $47 \%$ achieved a SDS global score $\leq 5$ (indicative of a normative range) compared to $28 \%$ of placebo treated patients. Duloxetine treated patients reported a greater improvement in their quality of life, as measured with the Quality of Life Enjoyment and Satisfaction Questionnaire Short Form (Q-LES-Q-SF), than did placebo treated patients $(\mathrm{p}<0.01$; ANOVA). The data suggest that not only symptomatic improvements are achieved by patients treated with duloxetine but that these improvements impact on social function and life satisfaction.

\section{Safety and tolerability}

\section{Treatment-emergent side effects}

Based on the pooled analysis of the three placebo controlled trials, discontinuation rates in patients receiving duloxetine due to adverse events was $15.6 \%$ compared to $4.2 \%$ for placebo ( $p<0.001$ ) (Allgulander et al 2007). The most common side effects $(>5 \%$ incidence and twice the rate in placebo treated patients) were nausea, dizziness, dry mouth, constipation, insomnia, somnolence, hyperhidrosis, decreased libido, vomiting and erectile dysfunction. For the majority of patients these side effects were rated as mild to moderate in intensity. These adverse events are similar to those reported in pooled analyses for the use of duloxetine in other conditions. For example, the tolerability of duloxetine, when used as a treatment for depression, was addressed in a pooled analysis from eight placebo controlled comparisons (Hudson et al 2005). The data base represented 1139 patients treated with doses of duloxetine ranging from 40 to $120 \mathrm{mg}$ /day for up to 34 weeks in total. Nausea, dry mouth, constipation, insomnia, dizziness and fatigue were the main side effects reported in these studies and all occurred significantly more frequently than with placebo. Cardiovascular assessments showed a slight increase in systolic and diastolic blood pressure as well as heart rate compared to placebo but were unlikely to be clinically significant. The incidence of abnormal laboratory parameters was generally similar between placebo and duloxetine treated patients. Most notably there were increases in liver enzyme values in patients treated with duloxetine, but the changes were not regarded as clinically important. There were 3 deaths in duloxetine-treated patients: 1 due to suicide, 1 due to cardiorespiratory arrest and the other to 
non-cardiogenic pulmonary edema. A series of 4 case reports suggesting a close temporal relationship between duloxetine use and the emergence of suicidal ideation in adult patients has been reported (Parikh et al 2008). In each of the cases suicidal ideation emerged in the context of increasing the dose of duloxetine. These ideas resolved when duloxetine was stopped.

Another pooled analysis was performed on the tolerability data for 23,983 patients exposed to duloxetine in 64 studies for any indication, including GAD (Gahimer et al 2007). The most common treatment emergent adverse events were nausea, headache, dizziness, dry mouth, constipation, insomnia, somnolence, hyperhidrosis, diarrhea and fatigue. These effects were reported to emerge early in treatment and were of mild to moderate severity. Generally they were tolerated with time. Increases in systolic and diastolic blood pressure on average were $<1 \mathrm{mmHg}$.

It can be concluded for these analyses that duloxetine at the doses used results in side effects consistent with the known pharmacological profile of the drug. In general the compound is well tolerated by most patients, at least in the short term. Some information on long term safety is available from studies in depression. An open label extension of duloxetine in doses of 60 to $120 \mathrm{mg}$ /day was conducted in 81 patients with major depressive disorder for up to two years of treatment (Wohlreich et al 2007). Treatment emergent side effects included upper respiratory tract infection (13.1\%), headache $(10.7 \%)$, insomnia $(10.7 \%)$, anxiety $(9.5 \%)$, weight gain $(9.5 \%)$, nasopharyngitis $(8.3 \%)$, constipation $(7.1 \%)$, hyperhidrosis $(7.1 \%)$ and abnormal dreams $(6.0 \%)$. Both diastolic and systolic blood pressure were increased by about $1 \mathrm{mmHg}$ and heart rate was increased by a mean of about $3 \mathrm{bpm}$. The data suggest that duloxetine is not associated with major side effects on long term administration. Nevertheless the database is relatively small. Further data on the longer term use of the drug and its safety and tolerability in patients with GAD are required.

\section{Cardiovascular and electrocardiographic effects}

Of growing concern in the use of many medications has been the risk of sudden cardiac death associated with prolongation of the corrected QT interval (QTc) (Fermini and Fossa 2003). The effects of supra-therapeutic exposures to duloxetine were evaluated in 117 healthy female volunteers using a randomized, double-blind, placebo controlled, cross-over study (Zhang et al 2007). Subjects received placebo for 22 days or duloxetine for 20 days with a 14 day washout period between regimens. In addition each subject received a single oral dose of $400 \mathrm{mg}$ moxifloxacin before or after the placebo/duloxetine treatment as a positive control. Duloxetine was administered to a maximum dose level of $200 \mathrm{mg}$ twice daily using a step-wise dose escalation procedure. ECG was recorded at four time points $(2,6,10$ and 12 hours) at baseline, fourth day of duloxetine dosing, and at the two highest dose levels (160 mg bd and $200 \mathrm{mg}$ bd). Following moxifloxacin ECG was recorded 2 and 6 hours after dosing. Data were analyzed using 3 QT interval correction methods: mixed effects analysis of covariance with RR interval change from baseline as the covariate, Fridericia's correction method and an individual QT correction method. The mean QTc interval was not prolonged for any of the three correction methods. On the contrary mean QTc interval decreased from baseline. Moxifloxacin prolonged QTc at all time points regardless of the correction method. Duloxetine at supra-therapeutic doses is unlikely to affect the QT interval in healthy subjects. These findings are consistent with the data obtained in clinical trials where mean change in the QRS width of the electrocardiogram was judged not likely to be of clinical significance (Hudson et al 2005). A further evaluation of the cardiovascular safety of duloxetine was performed based on all patients exposed to the drug in clinical trials up to December 2005 (Wericke et al 2007). A total of 8504 patients from 42 trials were included in the analysis. The safety profile was based on vital signs, ECGs and emergent events potentially related to cardiovascular effects of the drug. Duloxetine treated patients were more likely than placebo treated patients to have increases in heart rate and decreases in QTc (Fridericia's correction) interval. However none of the changes observed were regarded as clinically significant. Only one duloxetine treated patient had a prolongation of the QTc interval to $>500$ msecs (from 499 msecs at baseline to 514 msecs at maximum post baseline observation). In duloxetine-treated patients mean increase in systolic and diastolic blood pressure was $0.65 \mathrm{mmHg}$ and $0.88 \mathrm{mmHg}$ respectively. There was no evidence for a sustained increase in blood pressure associated with the use of duloxetine. Cardiovascular-related adverse events such as palpitations, tachycardia, orthostatic hypotension, hypertension and peripheral edema occurred in duloxetine treated patients to about the same extent as in placebo treated patients. With the exception of palpitations ( $1.5 \%$ for duloxetine treated patients) these events occurred with a frequency less than $1 \%$.

\section{Discontinuation events}

In the placebo controlled studies evidence for discontinuation effects following cessation of duloxetine was sought 
during a two-week tapering of the medication. There was no difference in the proportion of patients experiencing any event between duloxetine and placebo groups (Allulgander et al 2007). Dizziness, headache and insomnia all occurred more frequently with duloxetine, whereas upper respiratory infection occurred more frequently with placebo. In the trials discontinuation events were noted in $19.4 \%$ to $31.1 \%$ of duloxetine-treated patients and $15.8 \%$ to $17.3 \%$ of placebotreated patients. In none of the trials was the difference between duloxetine and placebo statistically significant. There did not appear to be any difference in the frequency of withdrawal symptoms depending on the dose of duloxetine.

Following abrupt discontinuation in depression trails there was no evidence of a severe withdrawal syndrome (Hudson et al 2005). Despite this it is recommended that the drug be tapered on withdrawal from treatment.

\section{Conclusions}

These studies in relatively large populations of patients provide reasonable evidence of the efficacy of duloxetine as a short term treatment for GAD. There were significant reductions in the baseline scores in the HAM-A scale during treatment for up to 10 weeks with the drug. Some $50 \%$ to $60 \%$ of patients achieved a $>50 \%$ reduction in their initial scores. In addition to symptomatic relief significant improvements in role functioning and quality of life were also attained by patients treated with duloxetine in comparison to placebo. At the doses used the drug is associated with some side effects which are generally predictable based on the known pharmacology of the agent. On the whole the side effects were of mild to moderate severity and were well tolerated, tending to diminish in intensity over time. With respect to cardiovascular safety a specific study evaluated effects on the QT interval at doses above those recommended for treatment of GAD and found no clinically significant prolongation of the interval. However this study was conducted in otherwise healthy volunteers and does not ensure the cardiac safety in patients with an already compromised system (eg, previous myocardial infarction, heart failure). Concerns about a potential withdrawal syndrome concluded that there may be a mild syndrome provided the drug is tapered during discontinuation. Abrupt withdrawal may also be a safe procedure, but there is little data with which to confidently assert this conclusion. Clearly, further work is necessary to address the issues of duloxetine long term safety and efficacy, at least for patients with a diagnosis of GAD.

Of note with all of the papers reviewed here is the potential for a conflict of interest of the authors. A recent analysis has suggested that both industry sponsorship and author conflict of interest affect study outcomes (Perlis et al 2005). While potential conflicts of interest do not imply unethical behavior or wrongdoing, the possibility of the publication of more favorable results exists. In the studies reviewed there is a noticeable involvement of employees of the manufacturer of duloxetine in the authorship of the papers. It is not clearly delineated what role these particular authors had in the final content of the papers and whether that involvement extended beyond statistical advice and provision of the raw data. This is not to imply that papers reviewed were unsound, either from the clinical or statistical methodology used, on the contrary this was exemplary. While ideally clinical evaluations of medicines should be conducted independently of the manufacturer it is recognized that costs of clinical trials are now beyond the scope of individual investigators. Nevertheless, for various reasons, the issues of financial conflict of interest have been shown to influence the reporting of such trials (Healy and Cattell 2003). Furthermore, when published clinical trial data are compared with that held in databases which include unpublished data there is a tendency for the efficacy of drugs to be inflated (Turner et al 2008). We are not aware of any unpublished or failed trials of duloxetine in GAD at the time of publication which might influence the conclusions about the efficacy of drug.

\section{Disclosures}

Neither author declares any potential conflict of interest with respect to this publication.

\section{References}

Allgulander C, Hartford J, Russell J. 2007. Pharmacotherapy of generalized anxiety disorder: results of duloxetine treatment from a pooled analysis of three clinical trials. Curr Med Res Opin, 23:1245-52.

Allgulander C, Nutt D, Detke M, et al. 2008. A non-inferiority comparison of duloxetine and venlafaxine in the treatment of adult patients with generalized anxiety disorder. $J$ Psychopharmacol, 22:417-25.

Anderson I. 2006. The new guidelines from the British Association for Psychopharmacology for anxiety disorders. Int J Psychiatr Clin Pract, 10:10-7.

Baldwin DS. 2006. Serotonin noradrenaline reuptake inhibitors: A new generation of treatment for anxiety disorders. Int J Psychiatr Clin Pract, 10:12-5.

Baldwin DS, Polkinghorn C. 2005. Evidence-based pharmacotherapy of generalized anxiety disorder. Int J Neuropsychopharmacol, 8:293-302.

Ballenger JC. 2001. Overview of different pharmacotherapies for attaining remission in generalized anxiety disorder. J Clin Psychiatry, 62:11-9.

Borsini F, Podhorma J, Marazzitti D. 2002. Do animal models of anxiety predict anxiolytic-like effects of antidepressants. Psychopharmacology, 163:121-41.

Bymaster FP, Dreshfield-Ahmad LJ, Threlkeld PG, et al. 2001. Comparative affinity of duloxetine and venlafaxine for serotonin and norepinephrine transporters in vitro and in vivo, human serotonin receptor subtypes and other neuronal receptors. Neuropsychopharmacology, 25:871-80. 
Bymaster FP, Lee TC, Knadler MP, et al. 2005. The dual transport inhibitor duloxetine: A review of its preclinical pharmacology, pharmacokinetic profile and clinical results in depression. Curr Pharm Des, 11:1475-93.

Carter RM, Wittchen H-U, Pfisster H, et al. 2001. One-year prevalence of subthreshold and threshold DSM-IV generalised anxiety disorder in a nationally representative sample. Depress Anxiety, 13:78-88.

Chan C, Yeo KP, Pan AX, et al. 2007. Duloxetine pharmacokinetics are similar in Japanese and Caucasian subjects. Br J Clin Pharmacol, 63:310-14.

Davidson JR. 2001. Pharmacotherapy of generalised anxiety disorder. J Clin Psychiatry, 62(Suppl 11):46-50.

Davidson JR, Allqulander C, Pollack MH, et al. 2008. Efficacy and tolerability of duloxetine in elderly patients with generalized anxiety disorder: a pooled analysis of four randomized, double-blind, placebo-controlled studies. Hum Psychopharmacol, 23:519-23.

Dunner DL, Goldstein DJ, Mallinckrodt C, et al. 2003. Duloxetine in the treatment of anxiety symptoms associated with depression. Depress Anxiety, 18:53-61.

Durham R, Chambers JA, Power KG, et al. 2005. Long-term outcome of cognitive behaviour therapy clinical trials in Scotland. Health Technol Assess, 9:1-174.

Durham RC, Murphy T, Allan T, et al. 1994. Cognitive therapy, analytic psychotherapy and anxiety management training for generalised anxiety disorder. Br J Psychiatry, 165:315-23.

EMEA. ICH Topic E9: Statistical principles for clinical trials (CPMP/ ICH/363/96), 1998.

Endicott J, Russell JW, Raskin J, et al. 2007. Duloxetine treatment for role functioning improvement in generalized anxiety disorder: Three independent studies. J Clin Psychiatry, 68:518-24.

Engleman ES, Perry KW, Mayle DA, et al. 1995. Simultaneous increases of extracellular monoamines in microdialysates from hypothalamus of conscious rats by duloxetine, a dual serotonin and norepinephrine uptake inhibitor. Neuropsychopharmacology, 12:287-95.

Fermini B, Fossa AA. 2003. The impact of drug-induced QT interval prolongation on drug discovery. Nat Rev Drug Discov, 2:439-47.

Gahimer J, Wernicke J, Yalcin I, et al. A retrospective pooled analysis of duloxetine safety in 23,983 subjects. Curr Med Res Opin, 23:175-84.

Goldstein DJ, Lu Y, Detke MJ, et al. 2004. Duloxetine in the treatment of depression: a double-blind placebo-controlled comparison with paroxetine. J Clin Psychopharmacol, 24:389-99.

Goldstein DJ, Mallinckrodt C, Lu Y, et al. 2002. Duloxetine in the treatment of major depressive disorder: a double-blind clinical trial. $J$ Clin Psychiatry, 63:225.

Gorman JM. Treatment of generalized anxiety disorder. J Clin Psychiatry, 63:17-23.

Gould RA, Otto MW, Pollack MH, et al. 1997. Cognitive behavioural and pharmacological treatment of generalised anxiety disorder: a preliminary meta-analysis. Behav Res Ther, 28:285-305.

Healy D, Cattell D. 2003. Interface between authorship, industry and science in the domain of therapeutics. Br J Psychiatry, 183:22-7.

Hidalgo RB, Tupler LA, Davidson JRT. 2007. An effect size analysis of pharmacologic treatments for generalised anxiety disorder. $J$ Psychopharmacol, 21:864-72.

Hudson JI, Wohlreich MM, Kajdasz DK. 2005. Safety and tolerability of duloxetine in the treatment of major depressive disorder: analysis of pooled data from eight placebo-controlled clinical trials. Hum Psychopharmacol, 20:327-41.

Kasamo K, Blier P, de Montigny C. 1996. Blockade of serotonin and norepininephrine uptake processes by duloxetine: in vitro and in vivo studies in the rat brain. J Pharmacol Exp Ther, 277:278-86.

Kessler RC, Chiu TW, Demler O, et al. 2005. Prevalence, severity and comorbidity of 12-month DSM-IV disorders in the National Comorbidity Survey Replication. Arch Gen Psychiatry, 62:617-27.

Kingdon D, Tyrer P, Seivewright N, et al. 1996. The Nottingham Study of Neurotic Disorder: influence of cognitive therapists on outcome. $\mathrm{Br} J$ Psychiatry, 169:93-7.
Koponen H, Allgulander C, Erickson J, et al. 2007. Efficacy of duloxetine for the treatment of generalized anxiety disorder: Implications for primary physicians. Prim Care Companion J Clin Psychiatry, 9:100-7.

Lam RW. 2006. Generalized anxiety disorder: how to treat and for how long? Int J Psychiatr Clin Pract, 10:10-15.

Lantz RJ, Gillespie TA, Rash TJ, et al. 2003. Metabolism, excretion and pharmacokinetics of duloxetine in healthy human subjects. Drug Metab Dispos, 31:1142-50.

Lieb R, Becker E, Altamura C. 2005. The epidemiology of generalized anxiety disorder in Europe. Eur Neuropsychopharmacol, 15:445-52.

Lieb R, Isenee B, von Sydow K, et al. 2000. The early stages of psychopathology study (EDSP), a methodological update. Eur Addict Res, 6:170-82.

NICE. Clinical Guideline 22. 2004. Anxiety: Management of anxiety (panic disorder, with or without agoraphobia and generalised anxiety disorder) in adults in primary, secondary and community care. URL: www.nice. orh.uk/CG022NICEguideline.

Nicolini H, Bakish D, Duenas H, et al. 2008. Improvements of psychic and somatic symptoms in adult patients with genearalised anxiety disorder: examination froma a duloxetine, venlafaxine extended release and placebo trial. Psychol Med, doi:10.1017/S0033291708003401.

Parikh AR, Thatcher BT, Tamano EA. 2008. Suicidal ideation associated with duloxetine use. J Clin Psychopharmacol, 28:101-2.

Perlis RH, Perlis CS, Wu Y, et al. 2005. Industry sponsorship and financial conflict of interest in the reporting of clinical trials in psychiatry. Am J Psychiatry, 162:1957-60.

Rynn M, Russell J, Erickson J, et al. 2007. Efficacy and safety of duloxetine in the treatment of generalized anxiety disorder: A flexible dose, progressive-titration, placebo controlled trial. Depress Anxiety, 22:1-8

Shader RI, Greenblatt DJ. 1993. Use of benzodiazepines in anxiety disorders. $N$ Engl J Med, 328:1398-405.

Sharma A, Goldberg MJ, Cerimele BJ. 2000. Pharmacokinetics and safety of duloxetine, a dual-serotonin and norepinephrine reuptake inhibitor $J$ Clin Pharmacol, 40:161-7.

Skinner MH, Kuan Han-Yi, Skerjanec A, et al. 2003. Effect of age on the pharmacokinetics of duloxetine in women. Br J Clin Pharmacol, 57:54-61.

Skinner MH, Skerjanec A, Seger M, et al. 2000. The effect of food and bedtime administration on duloxetine pharmacokinetics. Clin Pharmacol Ther, 67:129.

Stahl SM, Grady MM, Moret C, et al. 2005. SNRIs: Their pharmacology, clinical efficacy and tolerability in comparison with other classes of antidepressants. CNS Spectr, 10:732-47.

Suri A, Reddy S, Gonzales C, et al. 2005. Duloxetine pharmacokinetics in cirrhotics compared with healthy subjects. Int J Clin Pharmacol Ther, 43:78-84.

Tianmei S, Knadler MP, Lim MT, et al. 2007. Pharmacokinetics and tolerability of duloxetine following oral administration to healthy Chinese subjects. Clin Pharmacokinet, 46:767-5.

Troelsen KB, Nielsen EO, Mirza NR. 2005. Chronic treatment with duloxetine is necessary for an anxiolytic-like response inthe mouse zero maze: the role of the serotonin transporter. Psychopharmacology, 181:741-50.

Turcotte JE, Debonnel G, de Montigny C, et al. 2001. Assessment of the serotonin and norepinephrine reuptake blocking properties of duloxetine in healthy subjects. Neuropsychopharmacology, 24:511-21.

Turner EH, Matthews AM, Lindardatos E, et al. 2008. Selective publication of antidepressant trials and its influence on apparent efficacy. $N$ Engl $J \mathrm{Med}, 358: 252-60$.

Tyrer P, Baldwin D. 2006. Generalised anxiety disorder. Lancet, $368: 2156-64$

Vincent S, Bieck PR, Garland EM, et al. 2004. Clinical assessment of norepinephrine transporter blockade through biochemical and pharmacological profiles. Circulation, 109:3202-7.

Wernicke J, Lledo A, Raskin J, et al. 2007. An evaluation of the cardiovascular safety profile of duloxetine: findings from 42 placebo-controlled studies. Drug Saf, 30:437-55. 
Wittchen H-U, Carter RM, Pfisster H, et al. 2000. Disabilities and quality of life in pure and co-morbid generalised anxiety disorder and major depression in a national survey. Int Clin Psychopharmacol, 15:319-28.

Wittchen H-U, Nelson CB, Lachner G. 1998. Prevalence of mental disorders and psychosocial impairment in adolescents and young adults. Psychol Med, 28:109-26.

Wohlreich MM, Mallinckrodt CH, Prakash A, et al. 2007. Duloxetine for the treatment of major depressive diosrder: safety and tolerability associated with dose escalation. Depress Anxiety, 24:41-52.
Wong DT. 1998. Duloxetine (LY248686): an inhibitor of serotonin and noradrenaline uptake and an antidepressant drug candidate. Exp Opin Investig Drugs, 7:1-9.

Yonkers KA, Dyck IR, Warshaw M, et al. 2000. Factors prediciting the course of generalised anxiety disorder. Br J Psychiatry, 176:544-9.

Zhang L, Chappell J, Gonzales CR, et al. 2007. QT effects of duloxetine at supratherapeutic doses: a placebo and positive controlled study. J Cardiovasc Pharmacol, 49:146-53. 